Rotational Particle Separator:

\title{
A New Method for Separating Fine Particles and Mists from Gases
}

\author{
Bert Brouwers *
}

An account is given of the patented technique of the rotational particle separator for separating solid and liquid particles of diameter $0.1 \mu \mathrm{m}$ and larger from gases. Attention is focussed on the working principle, fluid mechanical constraints, practical designs, separation performance, power consumption, dimensions, filter cleaning and costs. Furthermore, an overview is given of current developments to introduce this technique in industrial dust removal, in electricity generation and in the consumer goods market. The article concludes with a summary of the features which distinguish this technique from existing filtering methods.

\section{Introduction}

Several techniques are available for the separation of fine particles from gases: these include use of electrostatic precipitators, baghouse filters and wet scrubbers. Each of these techniques has its advantages and disadvantages, and a choice is made depending on the requirements of the application. For an overview of existing filtering techniques the rẹader is referred to Dorman [1], Stern [2] and Löffler [3].

This article describes a new method for separating fine particles from gases. It concerns the patented technique of the rotational particle separator for separating solid and/or liquid particles of diameter $0.1 \mu \mathrm{m}$ and larger from gases [4, 5]. An account is given of working principle, designs, separation performance, power consumption, costs and current state of development of the technique.

\section{Working Principle}

The core component of the rotational particle separator is the filter element (see Fig. 1). It consists of a multitude of axial channels which rotate as a whole around a common rotational axis. The channels can have many cross-sectional forms, which share the common feature that each channel is enclosed by a single continuous wall. Each channel is bounded by a wall in radial as well as tangential direction. In case of channels formed by concentric cylinders each channel is partitioned by at least one radially extending wall (Fig. 1-IV).

Solid and/or liquid particles present in the gas flowing through the channels are centrifuged towards the outer collecting walls (see Fig. 2). As a result of the action of centrifugal forces, van der Waals' forces and/or forces due to surface tension, particles remain at the wall. The purified gas

\footnotetext{
* Prof. Dr. Ir. Bert Brouwers, Thermal Engineering, Twente University of Technology, P.O. Box 217, 7500 AE Enschede, The Netherlands.
}

leaves the channels while the particulate material collected at the walls can be removed periodically, for example by passing gas or liquid at high velocity through the channels.

The radial height of the channels is small (up to a few millimetres), so that the radial distance to be travelled by a particle is small. At limited circumferential speed of the filter element (some tens of metres per second) and relatively large axial gas velocities (a few metres per second) particles of (sub)micron size can still reach the outer collecting wall for sufficient axial length of the channels (up to about $1 \mathrm{~m}$ ). The diameter of the filter element can be up to $1 \mathrm{~m}$ allowing gas flows of $10,000 \mathrm{~m}^{3} / \mathrm{h}$. Length and height of the chan-

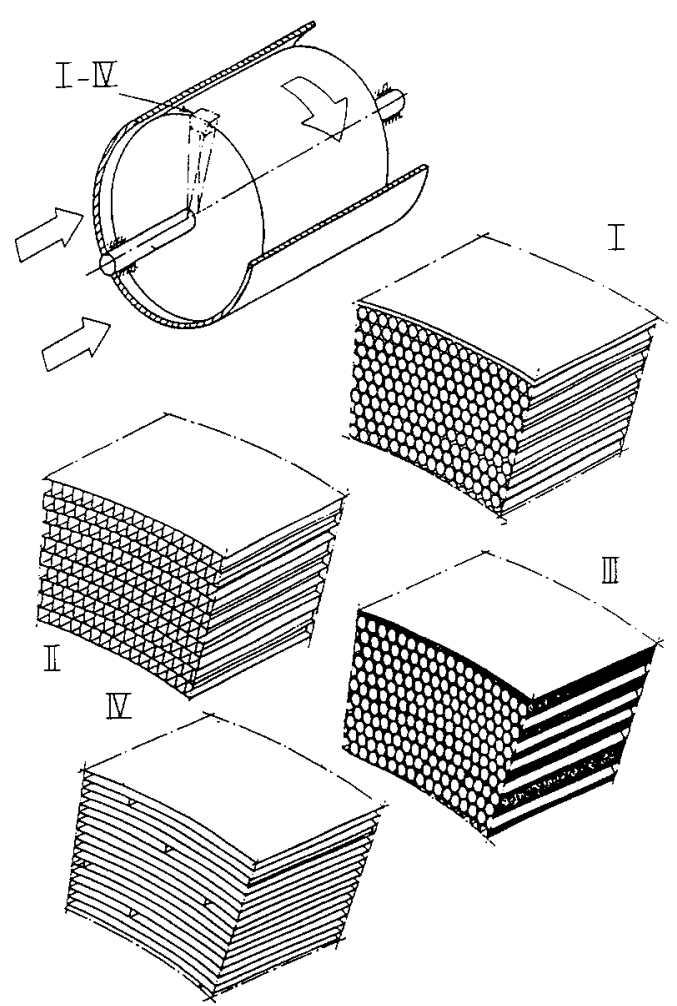

Figure 1. Filter element of the rotational particle separator. 


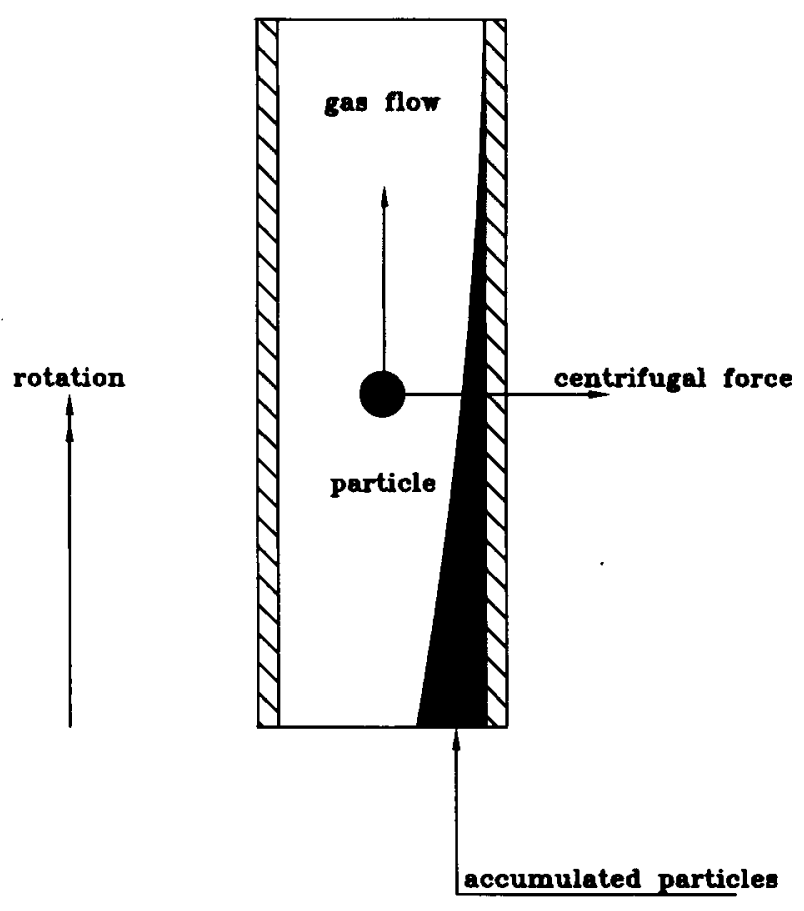

Figure 2. Centrifugally induced particle separation.

nels are dimensioned such that the pressure loss is limited to several hundred Pascal at most.

\section{Fluid Mechanical Constraints}

Although at first glance simple and straightforward, centrifugally induced migration of particles in the separation channels of the rotational particle separator is a subtle and sensitive process. The smallest particles separated move with radial velocities which are related to the axial gas velocity as the ratio of the height of the channel to its length. For practical versions of the rotational particle separator this ratio is as small as one in hundred, implying radial migration velocities of only a few centimetres per second. Furthermore, the channels rotate with circumferential speeds of some tens of metres per second. Under such circumstances, secondary gas flows in a plane perpendicular to the axial flow can easily develop, which disturb the slow radial migration of the particles. For optimum operation of the process, an almost purely axial gas flow in the channels is necessary.

The required almost purely axial flow in the channels is accomplished if the following conditions are satisfied:

(i) To obtain laminar flow, the channels have to be dimensioned such that the Reynolds number based on average axial gas velocitv is less than ahout 2000 .

(ii) The above requirement to ensure stability of laminar flow has been derived from flow in non-rotating channels. It is known that in case of rotation the value of the axial flow Reynolds number at which instability occurs can become much lower, i.e. by a value which can become about 30 times smaller (see Section 46 in [6] and [7]). This reduction and the resulting considerable reduction of axial gas flow can be prevented by satisfying the second condition, namely that each channel is enclosed by a single continuous wall. By bounding the channels in both the radial and the tangential direction, destabilisation of the flow due to rotation is counteracted. Under the flow conditions of practically dimensioned rotational particle separators, adopting channels with singly-connected cross-section satisfying condition (i) is sufficient to ensure laminar flow.

(iii) In practical applications of the rotational particle separator, the channels will not be entirely parallel to the rotation axis. In case of non-parallelity, Coriolis forces will act on the gas. These Coriolis forces in combination with Hagen-Poiseuille flow in the channels will lead to circulatory flows in the cross-sectional plane of the channels $[8,9]$. These secondary flows can disturb the centrifugally induced radial migration process of particles. The particle separation process becomes seriously affected for circulatory flow velocities which are of the same order of magnitude as the radial migration velocity, or larger [10]. This leads to the requirement that the degree of non-parallelity of the separation channels with respect to the rotation axis should be limited to a specific value [10].

The above requirements pursuant to the flow in rotating channels are essential for effective functioning of the rotational particle separator. Satisfying these requirements, on the other hand, does not pose a serious limitation for the practical applicability of the rotational particle separator.

\section{Practical Designs}

To meet the demands of specific applications, various designs of the rotational particle separator have been undertaken. These include the axial version and tangential version, where axial and tangential refer to the direction of the gas flow at the inlet.

\subsection{Axial Version}

In the case of the axial version shown in Fig. 3, gas containing particulate material is directed axially into an impeller. The impeller is fitted upstream of the filter element. In the impeller the contaminated gas is distributed over the channels of the filter element.

Another impeller is fitted downstream from the filter element. In this impeller, purified gas leaving the filter element is directed radially towards a volute. In this volute the gas is directed towards the outlet analogous to the functioning of a radial fan. Upper and lower impeller and filter element are connected to each other and are driven by a motor positioned in the centre.

An advantage of the axial version of the rotational particle separator is its compactness. The filter element, however, is 
not easily accessible for removing particulate material collected in the channels. The axial version is therefore suited for applications where the concentration of particles material in the gas is limited. The axial version is also suited for cases where the gas is contaminated with liquids. These liquid particles form a stable liquid film along the outer collecting walls of the channels, which flows downwards towards the lower impeller. On leaving the channels, the liquid is centrifuged towards the outer radial wall of the impeller. Small holes in the outer wall of the impeller and further provisions allow for the continuous transport of the separated liquid particles material out of the rotational particle separator.

\subsection{Tangential Version}

The tangential version of the rotational particle separator is shown in Fig. 4. The contaminated gas is directed tangentially into a cyclone-type inlet-housing. Here the coarse fraction of the particulate material is centrifuged towards the stationary walls to leave the apparatus via the particle outlet at the bottom. The gas is subsequently guided towards the rotating filter element within which the fine particles material is separated. A radial impeller is mounted downstream from the filter element which serves to bring the gas up to the desired pressure. The gas leaves the apparatus via the volute enclosing the impeller.

The blades of the impeller extend over a radius which is larger than the outer radius of the filter element. This permits gas or liquid to be blown into the channels of the filter element from a device mounted on top of the rotational separator. Particulate material collected in the channels can thus be removed, either during filter operation or during a period of shut-off or shut-down of the apparatus.

Impeller and filter element are connected to the driving shaft of a top-fitted motor flexibly (e.g. by rubber dampers) mounted on the housing. The impeller is designed such that an overpressure exists in the volute with respect to the cyclone. This prevents unwanted leakage of non-filtered gas in the cyclone to filtered gas in the volute. Large tolerances are allowed between housing and rotating element. Easy assembly and disassembly is accomplished by lifting the rotating element out of the housing via a flange at the top.

\section{Elementary Separation}

As a result of the centrifugal force, particles entrained in the gas will move radially in the separation channels of the rotational particle separator. The velocity at which particles move radially can be calculated on the basis of equilibrium between the centrifugal force and Stokes force. Stokes force describes the resistance due to relative motion between particle and gas. From the equilibrium, an expression is obtained for the radial velocity of a particle $u_{\mathrm{p}}$ which is given by

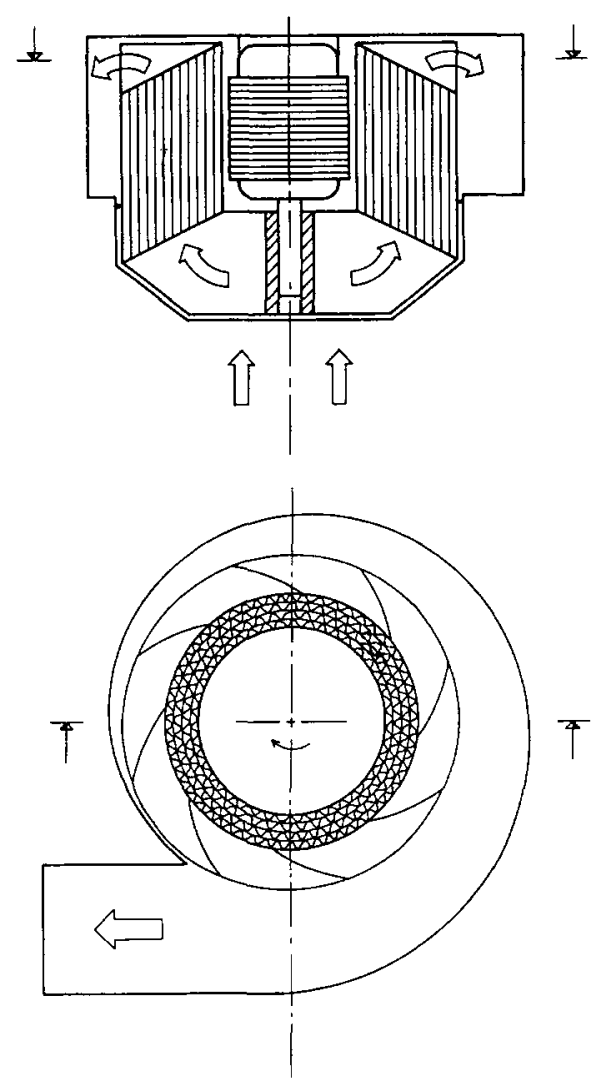

Figure 3. Axial version of the rotational particle separator.

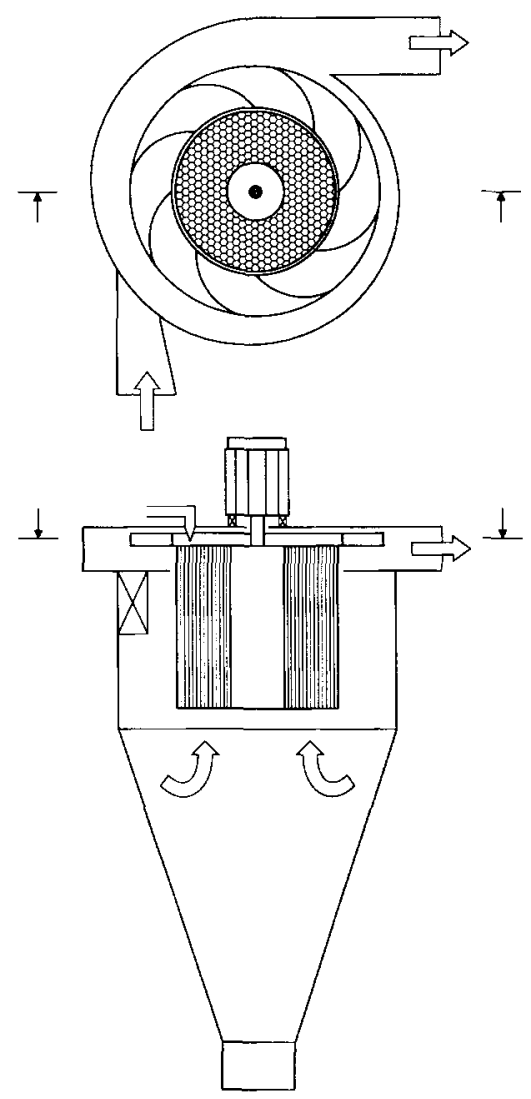

Figure 4. Tangential version of the rotational particle separator. 
$u_{\mathrm{p}}=\frac{\varrho_{\mathrm{p}} d_{\mathrm{p}}^{2} \Omega^{2} R}{18 \eta}$

where $\varrho_{\mathrm{p}}$ is the specific mass of the particle, $d_{\mathrm{p}}$ the particle diameter, $\Omega$ the angular velocity, $R$ the distance between channel and rotation axis, and $\eta$ the viscosity of the gas.

Whether a particle reaches the outer collecting wall of the channel depends on the residence time in the channel and the radial distance to be travelled by the particle. The smallest particle which is just able to reach the outer collecting wall with $100 \%$ probability is the particle which in the available time moves over a radial distance corresponding to the height of the channel. The radial velocity of this particle is

$u_{\mathrm{p}}=w_{\mathrm{gas}} d_{\mathrm{c}} / L$

where $w_{\text {gas }}$ is the axial gas velocity in the channel, $d_{c}$ the channel height and $L$ the channel length.

From Eqs (1) and (2) an expression can be derived for the smallest particle which is collected with $100 \%$ probability, i.e.

$d_{\mathrm{p} 100 \%}=\left(\frac{18 \eta w_{\mathrm{gas}} d_{\mathrm{c}}}{\varrho_{\mathrm{p}} \Omega^{2} R L}\right)^{1 / 2}$.

From this formula it is noted that for constant values of $d_{\mathrm{c}}$, $w_{\text {gas }}$ and $L, d_{\text {p } 100 \%}$ increases with decreasing distance $R$ from the rotation axis. This increase of $d_{\mathrm{p} 100 \%}$ can be avoided by designing an axial gas velocity $w_{\text {gas }}$ which increases linearly with distance $R$ from the rotation axis. The reduction in centrifugal force with decreasing $R$ is then compensated by an increase in residence time in the channel. The result is that the degree of particles separation will be the same for all channels. The desired distribution of the axial gas velocity can be accomplished by appropriate dimensioning of the inlet and outlet configuration upstream and downstream of the filter element.

In case of an optimum distribution of the axial gas velocity, i.e. $w_{\text {gas }} \div R$, an expression for $d_{\mathrm{p} 100 \%}$ can be derived which is applicable to the entire filter element:

$d_{\mathrm{p} 100 \%}=\left(\frac{27 \eta \phi d_{\mathrm{c}}}{\varrho_{\mathrm{p}} \Omega^{2} L \pi(1-\varepsilon)\left(R_{\mathrm{o}}^{3}-R_{\mathrm{i}}^{3}\right)}\right)^{1 / 2}$

where $\phi$ is the gas flow through the filter element, $\varepsilon$ the reduction of the effective cross-sectional area of the element due to wall thickness of the channels, $R_{\mathrm{o}}$ the outer radius of the element and $R_{\mathrm{i}}$ the inner radius.

Consider the following example:
$\phi=1 \mathrm{~m}^{3} / \mathrm{s}$
$L=0.6 \mathrm{~m} \quad R_{\mathrm{i}}=0.1 \mathrm{~m}$
$d_{\mathrm{c}}=2 \times 10^{-3} \mathrm{~m} \quad \varepsilon=0.1$
$\varrho_{\mathrm{p}}=2000 \mathrm{~kg} / \mathrm{m}^{3}$
$\Omega=150 \mathrm{rad} / \mathrm{s} \quad R_{\mathrm{o}}=0.3 \mathrm{~m}$
$\eta=1.8 \times 10^{-5} \mathrm{~kg} / \mathrm{ms}$

In this case one calculates:

$d_{\mathrm{p} 100 \%}=7 \times 10^{-7} \mathrm{~m}$

In other words, under the given conditions all particles with a diameter of $0.7 \mu \mathrm{m}$ and larger are separated in the rotating filter element considered. This result is obtained with a relatively small element: the residence time is less than $0.2 \mathrm{~s}$. Furthermore, the circumferential speed of the element is limited to $45 \mathrm{~m} / \mathrm{s}$, implying low friction and mechanical loading. According to the Hagen-Poiseuille formula for circular tubes [11], the pressure drop over the channels is assessed as $320 \mathrm{~Pa}$.

\section{Separation as a Function of Particle Size}

It follows from the previous considerations that collection rates of $100 \%$ can be expected for particles larger than $d_{\mathrm{p} 100 \%}$. Also particles smaller than $d_{\mathrm{p} 100 \%}$ can reach the outer collecting wall, provided that the distance of the particle from the wall when entering the separation channel is sufficiently small. In general the collection rate, i.e. the percentage of particles of a certain diameter which will reach the wall, will decrease with the degree by which these particles are smaller than $d_{\mathrm{p} 100 \%}$. The reduction of the collection rate will be a function of the ratio $d_{\mathrm{p}} / d_{\mathrm{p} 100 \%}$, where $d_{\mathrm{p}}$ is the particle diameter.

In Fig. 5 results obtained for collection efficiency are plotted versus particle diameter non-dimensionalised with $d_{\mathrm{p} 100 \%}$. Measurements were executed at various laboratories and involved the use of cascade impactors and laser particle counters. Data on test conditions and rotational particle separators used are presented in Tab. 1.

If present, deviations of particle collection efficiency shown in Fig. 5 can be ascribed to different distributions of the axial flow over the channels and measurement inaccuracies. It is furthermore observed that particles with a diameter larger

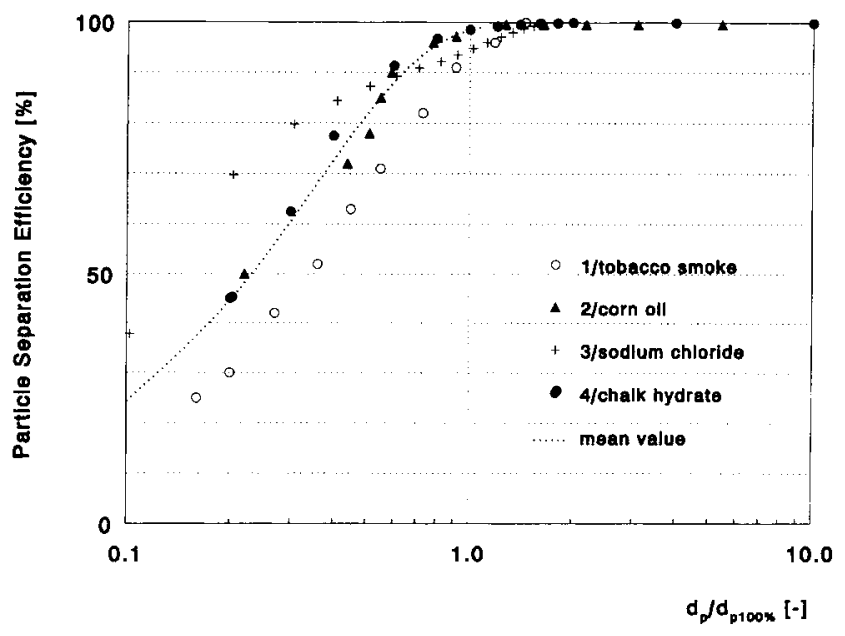

Figure 5. Particle collection efficiency versus dimensionless particle diameter measured with four rotational particle separators. 
Table 1. Data of tests with four rotational particle separators.

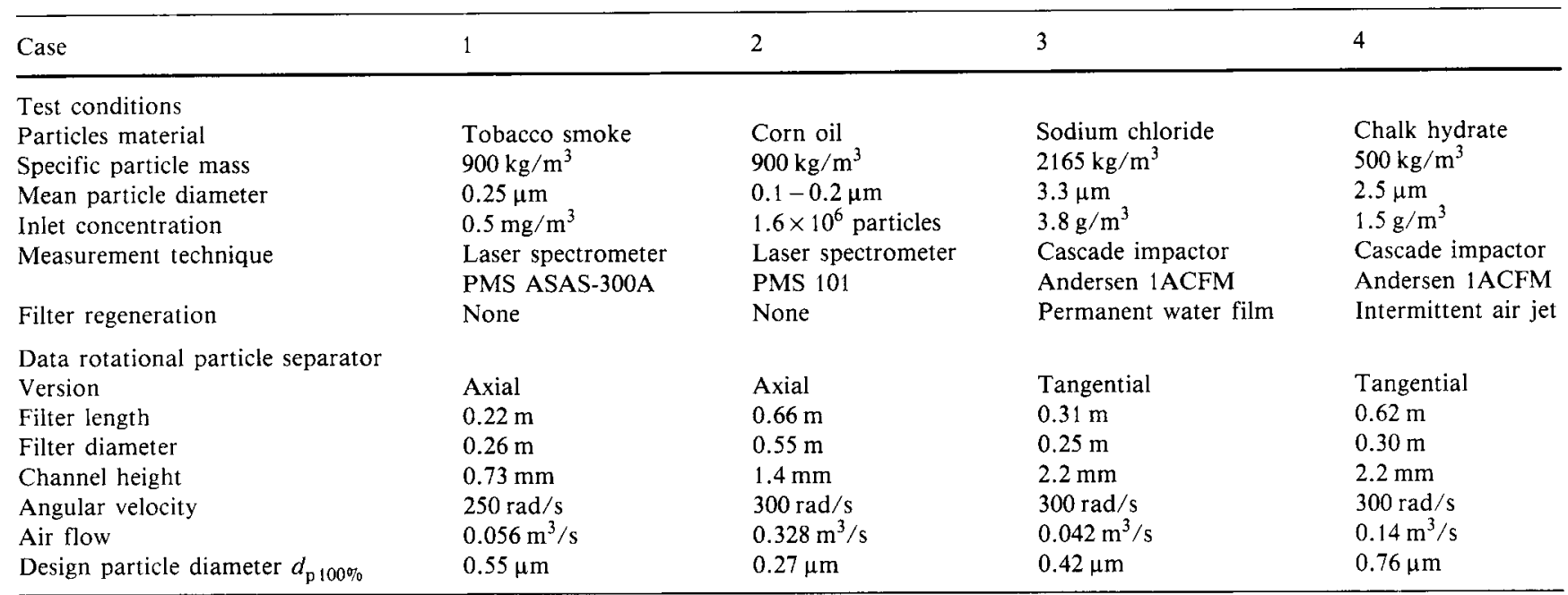

than $d_{\mathrm{p} 100 \%}$ are collected with practically $100 \%$ efficiency. In this connection it is noted that during the measurements with cascade impactors it was regularly found that practically no dust particles were present on the impactor stages corresponding to $d_{\mathrm{p}}>d_{\mathrm{p} 100 \%}$, despite high dust loads at the inlet. This result is attributable, among other things, to the fulfilment of the fluid mechanical conditions mentioned in Section 3.

As indicated earlier and apparent from Fig. 5, the particle collection efficiency decreases with decreasing particle diameter in the region $d_{\mathrm{p}}<d_{\mathrm{p} 100 \%}$. Also for wet scrubbers [12], particle collection efficiency decreases with decreasing particle diameter for sufficiently small values of $d_{\mathrm{p}}$. This is in contrast with the performance of electrostatic precipitators and baghouse filters, where it is found that, after an initial decrease, particle collection efficiency gradually increases with decreasing particle diameter [1-3]. This is ascribed to diffusional effects. These effects are also apparent in the channels of the rotational particle separator, but in practice for particle diameters less than approx. $0.01 \mu \mathrm{m}$. If considered advantageous, the performance of the rotational particle separator for ultra-fine particles can be enhanced (as alternative to reducing the value of $d_{\mathrm{p} 100 \%}$ ) by introducing means of congulating ultra-fine particles, e.g. electrostatic, turbulent or acoustic agglomeration devices, upstream of the filter element $[13,14]$.

On the basis of the measurements it is possible to construct a curve for the particle collection efficiency as a function of $d_{\mathrm{p}} / d_{\mathrm{p} 100 \%}$. This curve is shown as the dotted line in Fig. 5 which represents roughly the average of the plotted measurements. For a given distribution of the diameter of the particles at the inlet, this permits calculation of the overall collection efficiency with the design parameter $d_{\mathrm{p} 100 \%}$ as variable. As an illustration we can consider the case of particles whose diameter is represented by a log normal distribution. Such a distribution is quite common for aerosols originating from a single physical process/source [14]. For the geometrical standard deviation of the distribution we assume a value of 2.5 : i.e. $d_{\mathrm{p} 84} / d_{\mathrm{p} 50}=d_{\mathrm{p} 50} / d_{\mathrm{p} 16}=2.5$. In this case the overall collection efficiency to be expected from the rotational particle separator will only be a function of the ratio of the mean diameter of the particles $d_{\mathrm{pm}}$ and $d_{\mathrm{p} 100 \%}$. This dependence is shown in Fig. 6. It is seen that the overall collection efficiency increases with increasing $d_{\mathrm{pm}} / d_{\mathrm{p} 100 \%}$. For a rotational particle separator with a value of $d_{\mathrm{p} 100 \%}$ which is eight times smaller than the mean diameter of the particles entering the apparatus, an overall efficiency of $99.90 \%$ can be reached.

Summarising, the value of the design parameter $d_{\mathrm{p} 100 \%}$ as given by $\mathrm{Eq}$. (4) determines the separation performance of the rotational particle separator. The value of $d_{\mathrm{p} 100 \%}$ can be established by the magnitude of the gas flow, the channel height, the angular speed, the length and the radius of the filter element. By choosing proper values for these parameters designs can be realised which meet the requirements of the various applications.

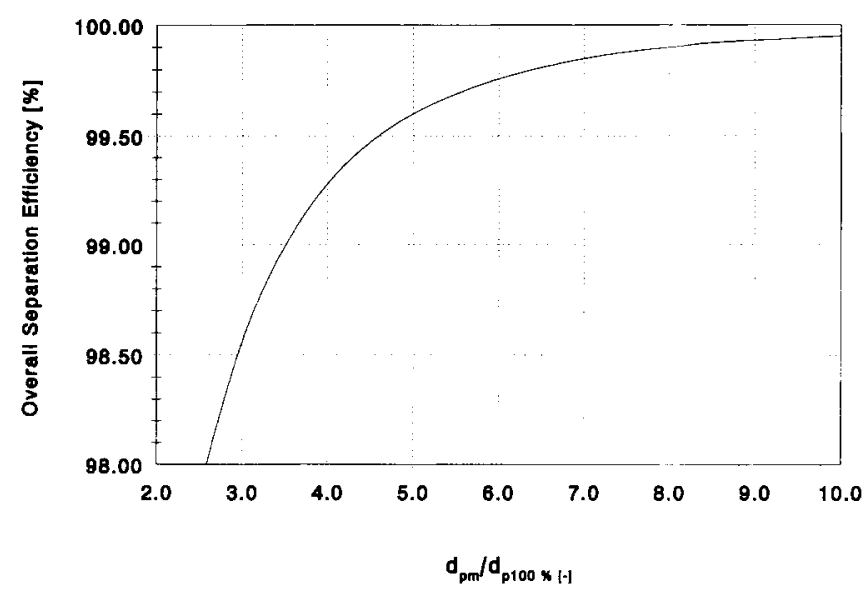

Figure 6. Overall collection efficiency versus ratio of the mean particle diameter $d_{\mathrm{pm}}$ and the design parameter $d_{\mathrm{p} 100 \%}$ in case of a log-normal distribution of the particles with $d_{\mathrm{p} 84} / d_{\mathrm{p} 50}=d_{\mathrm{p} 50} / d_{\mathrm{p} 16}=2.5$. 


\section{Power Consumption}

The power consumption of the rotational particle separator is mainly determined by irreversible pressure losses in the inlet housing, pressure drop over the separation channels, irreversible pressure losses in the volute and friction along the rotating walls. The total power consumption per unit of flow of gas to be purified, denoted by specific power consumption $p_{c}$, can be expressed by the following formula:

$p_{\mathrm{c}}=\frac{P_{\mathrm{s}}}{\phi}-\Delta p$,

where $P_{\mathrm{s}}$ is the shaft power, $\phi$ is the gas flow and $\Delta p$ is the pressure head delivered by the apparatus, i.e. pressure at outlet minus pressure at inlet. When the rotational particle separator is designed such that $\Delta p$ equals zero, i.e., when the impeller on top of the filter element generates a pressure head which just compensates for the internal pressure losses, the specific power consumption equals the shaft power divided by throughflow. The impeller, however, can also be designed such that the apparatus generates a net overpressure, for example to compensate for losses in other parts of a system of which the rotational particle separator forms a part. In this case, the shaft power will become higher.

Practical experience has shown that, on adopting formulae for flows and pressure losses in cyclones, channels, impellers and volutes, it is possible to predict shaft powers, pressures and velocity distributions over the channels of the filter element with reasonable accuracy as a function of design variables. For common designs the specific power consumption varies between 1000 and $2000 \mathrm{~J} / \mathrm{m}^{3}$. This value should be compared with the overall pressure loss of a baghouse filter divided by the efficiency of the fan which generates the flow. For electrostatic precipitators, it should be compared with the power requirements of the high voltage system and the pressure loss over the precipitator divided by the fan efficiency. For wet scrubbers, account must be taken of the pressure loss of the gas over the scrubber and the power required to pump the washing liquid.

The value of the specific power consumption $p_{\mathrm{c}}$ of the rotational particle separator depends on design variables. Designs aimed at collecting small particles can have larger values of $p_{\mathrm{c}}$ than those aimed at larger particles. Furthermore, the value of $p_{\mathrm{c}}$ decreases with increasing size of the apparatus, with the gas flow and separation performance being equal. For common designs the power consumption of the rotational particle separator is comparable with that of baghouse filters and electrostatic precipitators; it is much less than that of wet scrubbers.

\section{Dimensions}

The size or volume of the rotational particle separator per unit gas flow purified can be related to the residence time of the gas in the filter element. For common designs the res- idence time varies between 0.1 and $0.3 \mathrm{~s}$. Depending on type of design (Figs 3 and 4), the total volume of the apparatus is two to ten times the volume of the filter element.

Consider the rotating filter element treated as example in Section 5. This filter element has an outer diameter of $0.6 \mathrm{~m}$, a length of $0.6 \mathrm{~m}$ and is subjected to a flow of $1 \mathrm{~m}^{3} / \mathrm{s}$. Consider now the tangential version (Fig. 4) with a cyclone-type inlet housing which fits this filter element. The cyclone is designed such that the particle diameter separated with $50 \%$ probability amounts to about $5 \mu \mathrm{m}$. For a range of applications such as fly-ash of certain pulverizedcoal-fired combustion installations, cement kilns and waste incinerators, this will result in about $80 \%$ to $90 \%$ pre-separation of particles in the cyclone. To achieve this, the effective length of the cyclone should be about $1.5 \mathrm{~m}$. The external sizes of the apparatus are then: outer diameter approx. $1.1 \mathrm{~m}$, height measured from motor on top to particles exit at bottom approx. $2.5 \mathrm{~m}$. As stated above, the gas flow which is handled by this apparatus is $1 \mathrm{~m}^{3} / \mathrm{s}$. As calculated in Section 5, the value of $d_{\mathrm{p} 100 \%}$ equals $0.7 \mu \mathrm{m}$. On the basis of this value, the separation performance can be deduced from Figs 5 and 6 . The overall separation performance of this apparatus will be high for a range of applications and will meet stringent environmental legislation.

For larger throughflows of gas to be purified, use can be made of rotational particle separators arranged in parallel. Optimisation studies have shown that the throughflow of the single unit is limited to approx. 1 to $4 \mathrm{~m}^{3} / \mathrm{s}$. For larger flows, units are arranged in parallel. For both the axial and tangential version, it is possible to integrate the rotating elements in a joint housing, thus reducing costs. In case of the tangential version, integration can be accomplished in a manner analogous to that of multicyclones.

\section{Filter Cleaning}

For certain applications, filter cleaning involves removal of the filter element from the apparatus and followed by cleaning an reintroduction or by replacement. For most industrial applications, however, in-situ filter cleaning is preferred, without or with limited interruption of the filtering process. Three types of in-situ filter cleaning are considered: intermittent cleaning by air jets, intermittent cleaning by liquid jets and continuous cleaning by permanently wetting the filter element.

\subsection{Air Jet Cleaning}

A nozzle is fitted on top of the rotational particle separator which can move radially from inner to outer radius of the filter element: see Fig. 4. The nozzle is connected to a reservoir of compressed air, e.g. of 6 bar. Once the channels of the filter element become saturated with particulate material, the jet starts to blow into the channels. This can occur during normal filter operation. While the filter rotates, the nozzle moves radially with a speed such that each channel gets at least one blast of compressed air. 
The nozzle is positioned only a few millimetres above the filter element. The compressed gas comes out of the nozzle with supersonic speed, and recompresses upon entering the channels of the filter element, losing about $20 \%$ of its original pressure. In the channels a complex pattern of shock waves and expansion waves develops (see Fig. 7). The injected compressed air moves downwards with an initial velocity of about $280 \mathrm{~m} / \mathrm{s}$. In front of this injected air, a shock wave propagates downwards through the gas of originally 1 bar present in the channel. The propagation speed is initially about $570 \mathrm{~m} / \mathrm{s}$ and due to channel friction is reduced to about $470 \mathrm{~m} / \mathrm{s}$ upon arriving at the lower end of the channel. Behind this propagation wave, the gas is compressed to a density which is about 1.7 times the density of the undisturbed gas, and moves downwards with speeds of initially about $280 \mathrm{~m} / \mathrm{s}$ and at the bottom end of about 180 $\mathrm{m} / \mathrm{s}$. In this region of downward moving compressed air occurring in front of the air injected from the nozzle, particulate material is ripped off from the walls and transported downwards. The thickness of the layer of particulate material which moves downwards can be assessed from a balance between the shear force exerted by the gas and the friction between particles due to centrifugal pressure. It is found to be some tens of micrometres.

Due to filter rotation, a moment will occur when the channel has passed the column of air blown from the nozzle. At this moment expansion waves start to develop from the top of the channel, and the intensity of the axial momentum of the gas begins to decrease. Characteristically for supersonic conditions, the front of the expansion waves propagates faster than the compression wave. As cleaning occurs behind the front of the compression wave one should avoid a situation where the front of the expansion waves (line 2 in Fig. 7) catches up with the compression wave (line 1 in Fig. 7) before the latter arrives at the lower end of the channel. Therefore the jet should blow sufficiently long. This is achieved by designing the nozzle such that its length in the circumferential direction is sufficiently large, in practice a few centimetres. The radial width of the nozzle compares in size with the height of the channels, i.e. a few millimetres.

Under the above conditions, for practical versions of the rotational particle separator such as the rotational particle

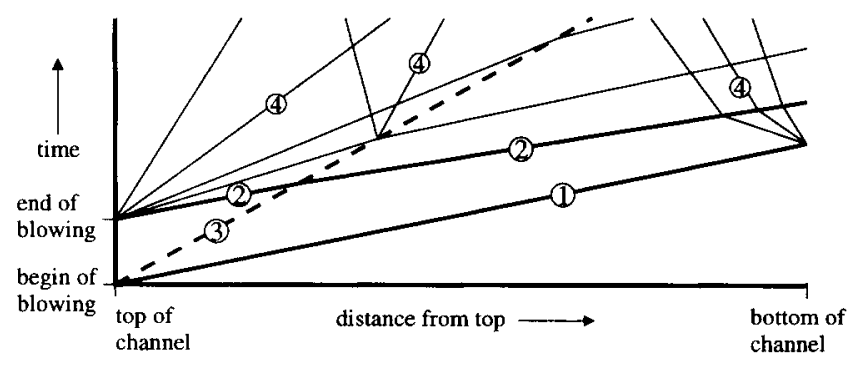

Figure 7. Pattern of shock, expansion and reflection waves in a separation channel due to air jet blowing at the top.

1. Shock wave in original gas.

2. Front of expansion waves through injected gas and original gas behind which the intensity of the axial momentum begins te decline.

3. Boundary between injected and original gas.

4. Expansion and reflection waves. separator fitted with the filter element treated as example in Section 5 , it has been established that about $1 \mathrm{~kg}$ of fine particles material collected in the channels can be removed by injecting about $1 \mathrm{~kg}$ of compressed air at 6 bar: see Fig. 8 . This result is obtained under normal operational conditions with the filter element under full rotation. It compares reasonably well with theoretical predictions based on previously described physics of shock waves and layers of particulate material being moved downwards. Taking account of pre-separation in the cyclone, for a range of practical applications a realistic value for the loading of the filter element is $1 \mathrm{~g}$ of fine particles material per unit of gas to be purified. The consumption of compressed air then amounts to about $0.8 \mathrm{Nm}^{3}$ for every $1000 \mathrm{~m}^{3}$ of gas to be purified. The power consumption of the compressor per unit of purified gas flow is then assessed as about $300 \mathrm{~J} / \mathrm{m}^{3}$. If required, these values of compressed air consumption and related power consumption can be reduced significantly by adopting a system in which the filter is taken off-line and subsequently cleaned at reduced rotational speed.

\subsection{Water Jet Cleaning}

As alternative to air (or other gases), cleaning of the filter element may be accomplished by periodically injecting water (or other liquids). In practice it has been established that (hot) water at pressures of 50 to 100 bar can be injected using the same nozzle as the one used for air. It of fers the possibility to clean from time to time the filter very thoroughly with (hot) water, in addition to a regular air cleaning. It is particularly interesting for applications where high standards of hygiene apply.

\subsection{Permanent Filter Wetting}

A third method for removing particles material from the channels of the filter element is to continuously add liquid. This can occur by dispersing a spray of fine liquid particles
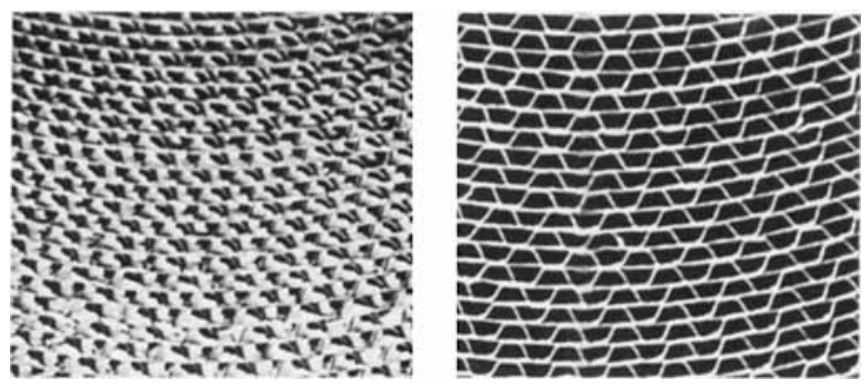

Figure 8. Photograph on the left shows part of the filter element of the rotational particle separator filled with separated particles material. Photograph on the right shows part of the same filter element after removal of particulate material. Removal was accomplished by blowing air at 6 bar pressure through a nozzle fitted on top of the rotational particle separator. The nozzle moves radially while the filter rotates ensuring that each channel is subjected to air shock. The filter element shown has been derived from the monolith of a catalytic converter made of high alloy stainless steel and has been tested at temperatures of up to $600^{\circ} \mathrm{C}$. 
in the gas upstream of the filter element. The fine liquid particles are centrifuged towards the outer walls of the channels of the filter element. Here they form a liquid film which moves downwards and which carries away the other (solid) particles. This technique has been successfully applied to both the axial and the tangential version of the rotational particle separator.

Another method for permanent wetting of the filter element involves addition of liquid from the top of the filter element. In practice this has been realised by using a small nozzle which moves slowly up and down in radial direction while the filter rotates. The desired amount of liquid (at 5 bar) is injected into the channels and forms a stable film along the outer walls of the channels. In this film centrifuged solid particles are collected, are dissolved or remain floating, and are transported downwards. The advantage of adding liquid from the top of the filter element is that the created liquid film extends over the entire length of the filter element.

The wet version of the rotational particle separator appears to be an attractive alternative to existing wet scrubbers often employed in the chemical and process industry. In contrast to wet scrubbers, in the rotational particle separator water is not injected to separate particles, but only to transport particles material being centrifuged towards the walls. This results in much lower (by up to two orders of magnitude) amounts of washing liquids. This is also one of the reasons why the power consumption is much less. For equal separation performance, the power consumption quoted for conventional wet scrubbers [12] is about five times larger than that established for the rotational particle separator.

\section{Manufacturing and Costs}

The principle of the rotational particle separator has a wide range of possible applications. Various development programs are in progress to prepare the technique for commercial application. These are described in the next section.

The costs of manufacturing the rotational particle separator are closely related to the kind of application. Costs can be limited as most components are common products: motor, housing (made of metal or plastic), impeller, equipment for filter cleaning, electronic controls, etc. The only component which is not a standard item is the filter element. A simple method of manufacturing this element follows the manufacturing process of corrugated board: Foil is corrugated and wound up together with non-corrugated foil. Foils are available relatively cheaply in thicknesses as small as $10 \mu \mathrm{m}$, the available thicknesses varying from plastic to aluminium and stainless steel.

For industrial applications, stainless steel filter designs are particularly interesting. To manufacture the filter element, use can be made of the manufacturing process of metallic monoliths for catalytic converters for car exhausts (Fig. 8).
These monoliths are manufactured according to the mentioned principles of corrugated board construction. The various layers of corrugated and non-corrugated metal foil are joined to each other by high-temperature vacuum brazing techniques [15]. Foils have thicknesses of $50 \mu \mathrm{m}$, consist of $\mathrm{Fe}-\mathrm{Cr}-\mathrm{Al}$ alloy and can withstand temperatures up to $1000^{\circ} \mathrm{C}$. On use of metal foils of thickness $50 \mu \mathrm{m}$, the weight of the filter element treated as example in Section 5 ( $D=0.6 \mathrm{~m}, L=0.6 \mathrm{~m}$ ) is calculated to be about $65 \mathrm{~kg}$.

Studies were carried out on the costs of filtering systems based on the rotational particle separator. Considering stainless steel designs suited for industrial applications, the discounted value of annual costs was assessed. These included purchasing costs, utility costs, costs of assembly and replacement, engineering costs and costs of construction and erection. For installations with a total gas flow up to about $100,000 \mathrm{~m}^{3} / \mathrm{h}$, it was found that the discounted value of annual costs, expressed in dollars per unit of gas flow being purified, were lower than those of existing filtering methods, in particular for installations with lower gas flows. In the case of baghouse filters and electrostatic precipitators, the cost advantage can be ascribed to lower costs for replacement and investment. For wet scrubbers, such as venturi scrubbers, the cost advantage is due to lower utility costs (lower power requirements).

\section{Overview of Current Applications and Developments}

The company Lebon \& Gimbrair (Bunschoten, The Netherlands) has started manufacturing rotational particle separators for source extraction and filtering of gases encountered in industrial processes: see Fig. 9. The market launch in The Netherlands started at the beginning of 1995. The apparatus is made of stainless steel. This offers important advantages for applications in, e.g., the pharmaceutical, food, chemical and dairy industries, where high standards of hygiene prevail, where recovery of product is desired, or where the particulate material to be collected changes regularly.

Together with an internationally operating company in the field of filters for welding fumes, Lebon \& Gimbrair has developed a prototype of the rotational particle separator suited for point extraction and filtering of welding fumes. External sizes of the prototype: height approx. $1 \mathrm{~m}$, diameter approx. $0.6 \mathrm{~m}$. Nominal flow is approx. $1200 \mathrm{~m}^{3} / \mathrm{h}$, while the design parameter $d_{\mathrm{p}^{100 \%}}$ normalised on a particle specific mass of $900 \mathrm{~kg} / \mathrm{m}^{3}$ amounts to $0.27 \mu \mathrm{m}$. The overall particle separation efficiency measured for welding fume particles (average diameter about $0.2 \mu \mathrm{m}$ ) amounted to around $90 \%$. The design will be adapted to meet recently introduced German standards of $98 \%$ separation efficiency. For this purpose, $d_{\mathrm{p} 100 \%}$ will be reduced, inter alia, by reducing the channel height of the filter element.

In cooperation with the chemical company Akzo Nobel, tests were carried out with a 'wet' version of the rotational particle separator at Twente University of Technology in The Netherlands. These tests involved the separation of fine 


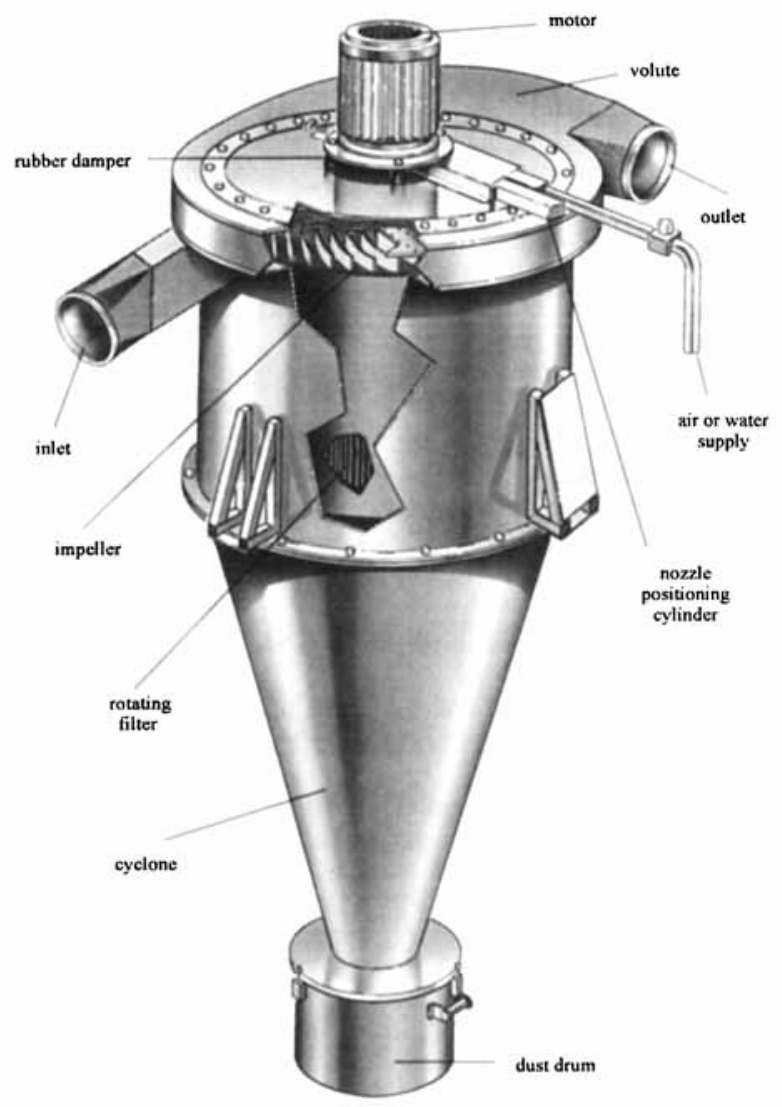

Figure 9. Rotational particle separator of stainless steel for source extraction, and filtering of gases of industrial processes with a throughput of up to $5000 \mathrm{~m}^{3} / \mathrm{h}$ (manufacturer Lebon \& Gimbrair, Bunschoten, The Netherlands). Jet-blowing of air and/or (hot) water through the nozzle fitted on top of the apparatus allows for the periodical removal of collected particles from the rotating filter element while the filter is in operation.

salt particles with an average diameter of $3.3 \mu \mathrm{m}$ dispersed in air at a concentration of $5 \mathrm{~g} / \mathrm{m}^{3}$. Overall collection efficiencies amounted to $99.85 \%$. The filter element was cleaned continuously by feeding water at the top of the filter element at a rate of 1 liter for every $50 \mathrm{~m}^{3}$ of air containing $0.25 \mathrm{~kg}$ of salt particles. A stable water film developed along the outer walls of the channels of the filter element in which the centrifuged salt particles dissolved and were carried downwards towards the outlet. The required amounts of washing water were about 100 times lower than those sprayed in wet scrubbers with similar performance, inter alia, venturi scrubbers. The power consumption was five times lower.

Another advantage of the rotational particle separator is that it can be used at high temperature. With suitably chosen materials, separation at temperatures of up to $1000^{\circ} \mathrm{C}$ is considered feasible. Supported by the Netherlands Ministry of Economic Affairs, successful tests were recently carried out on separation of residual particles from the flue gases of a fluidised bed combustor at a temperature of up to $600^{\circ} \mathrm{C}$ (Fig. 8). A further step in this development presently being taken is the use of the rotational particle separator as a reactor within which particles are dispersed to ab- sorb or react with gaseous contaminants present in the carrier gas.

The research institute of the joint electricity generating companies in The Netherlands, Kema, is presently conducting a development and testing programme aimed at using the rotational particle separator in electricity generation. One application concerns filtering intake air of gas turbines. After successful completion of tests on a prototype, the go-ahead was given to the erection of a test unit which is from May 1995 in operation and which consists of 4 rotational separators arranged in parallel with a total air throughput of $40,000 \mathrm{~m}^{3} / \mathrm{h}$. Both solid and liquid (mist) particles present in the air sucked from the environment are separated in this unit. Furthermore, the purified air is delivered at an overpressure. These factors are considered particularly advantageous for application to the filtering of intake air of gas turbines for permanent power generation.

At present, at the laboratory of Kema tests are being performed to separate fly-ash particles from the flue gases of a pulverised-coal-fired boiler with a gas throughput of $2000 \mathrm{~m}^{3} / \mathrm{h}$ at a temperature of $250^{\circ} \mathrm{C}$. Once these tests have been completed, this project will be continued with the erection of a scaled-up installation of rotational particle separators arranged in parallel and capable of filtering a slip-stream of about $40,000 \mathrm{~m}^{3} / \mathrm{h}$ taken from the flue gases of an existing coal fired electricity producing plant in The Netherlands. These projects form, inter alia, part of a program to develop techniques for purifying synthetic gases of coal gasification processes at high pressure and high temperature [16].

A multinationally operating company in the field of electric consumer products has developed a portable air cleaner embodying the principles of the rotational particle separator. The air cleaner is suited for purifying air in rooms, offices, etc., and is to be launched on the market at the end of this year.

\section{Concluding Remarks}

The features of the rotational particle separator can be summarised as follows:

- In practice, significant particle separation efficiencies (up to 100 percent) can be realised for particles of about $0.1 \mu \mathrm{m}$ in diameter and larger.

- Both solid and liquid particles can be separated simultaneously without disturbing the separation process; dry and wet dust removal are both possible.

- Filter cleaning can occur on-line, allowing continuous uninterrupted operation.

- Apparatus including the filter element can be designed for long lifetimes. It can be manufactured entirely from stainless steel and provided with means for cleaning with air and hot water.

- Application at high temperature, in a corrosive environment and at high pressure can be designed for. 
- Apart from single units, units can be arranged in parallel to purify large amounts of gas.

- The rotational particle separator has a built-in fan. Gases being purified can be fed at the desired pressure without an additional fan.

- The separation of particles is a well-defined and quantifiable physical process. For known particulate materials, separation and emission can be calculated in advance as a function of design parameters.

- Costs, sizes and power consumption can compete with those of existing filtering methods.

- Various development programs are taking place to demonstrate mechanical integrity and lifetime. For a number of applications market introduction has started or is pending.

\section{Acknowledgement}

The author would like to thank the Management of Romico Hold for permission to publish the information given in this article.

Received: December 5, 1994 [CET 746]

\section{References}

[1] Dorman, R.G., Dust Control and Engineering, Pergamon Press, Oxford 1974.

[2] Stern, A.C., Air Pollution, Volume IV: Engineering Control of Air Pollution, Academic Press, New York 1977.

[3] Löffler, F., Chem.-Ing.-Tech. 60 (1988) pp. $443-452$.

[4] Europ. Pat. 0.286.160 (1990) (Inv. J. J.H. Brouwers).

[5] US Patents 4,994,097 and 5,073,177 (1991) (Inv. J.J.H. Brouwers).

[6] Joseph, D.D., Stability of Fluid Motions. Springer Verlag, New York 1976.

[7] Mackrodt, P.-A., J. Fluid Mech. 73 (1976) pp. $153-166$.

[8] Barua, S. N., Proc. R. Soc. Lond. A227 (1954) pp. 133-139.

[9] Benton, G.S., J. Appl. Mech. 23 (1956) pp. $123-127$.

[10] Brouwers, J. J. H., Secondary Flows and Particle Centrifugation in Slightly Tilted Rotating Pipes, Appl. Sci. Res. 55 (1995) (to be published).

[11] Bird, R.B., Stewart, W.E., Lightfoot, E. N., Transport Phenomena, Wiley, New York 1960.

[12] Holzer, K., Chem.-Ing.-Tech. 51 (1979) pp. $200-207$.

[13] Wadenpohl, C., Löffler, F., Chem. Eng. Process. 33 (1994) pp. $371-377$.

[14] Hinds, W.C., Aerosol Technology. Wiley, New York 1982.

[15] Nonnenmann, M., Metal Supports for Exhaust Gas Catalysts, SAE paper 850131 (1985).

[16] Bolt, N., Proc. Ilth Ann. Int. Coal Conf., Pittsburgh (1994) pp. $1322-1327$. 